\title{
PPARס attenuates hepatic steatosis through autophagy-mediated fatty acid oxidation
}

\author{
Lei Tong ${ }^{1}$, Long Wang ${ }^{1}$, Shuangshuang Yao', Lina Jin', Jian Yang ${ }^{1}$, Yifei Zhang ${ }^{1}$, Guang Ning ${ }^{1}$ and Zhiguo Zhang ${ }^{1}$
}

\begin{abstract}
Peroxisome proliferator-activated receptor $\delta$ (PPAR $\delta$ ) belongs to the nuclear receptor family and is involved in metabolic diseases. Although PPARS is known to attenuate hepatic lipid deposition, its mechanism remains unclear. Here, we show that PPARS is a potent stimulator of hepatic autophagic flux. The expression levels of PPAR $\delta$ and autophagy-related proteins were decreased in liver tissues from obese and ageing mice. Pharmacological and adenovirus-mediated increases in PPAR $\delta$ expression and activity were achieved in obese transgenic $\mathrm{db} / \mathrm{db}$ and high fat diet-fed mice. Using genetic, pharmacological and metabolic approaches, we demonstrate that PPAR $\delta$ reduces intrahepatic lipid content and stimulates $\beta$-oxidation in liver and hepatic cells by an autophagy-lysosomal pathway involving AMPK/mTOR signalling. These results provide novel insight into the lipolytic actions of PPAR $\delta$ through autophagy in the liver and highlight its potential beneficial effects in NAFLD.
\end{abstract}

\section{Introduction}

Non-alcoholic fatty liver disease (NAFLD) is recognised as the leading cause of chronic liver disease in adults and children $^{1}$, with histological characteristics ranging from simple fatty liver (steatosis) to non-alcoholic steatohepatitis (NASH) and cirrhosis; some cases even develop into end-stage liver disease and hepatocellular carcinoma ${ }^{2,3}$. NAFLD appears to be highly associated with obesity and diabetes. NAFLD is characterised by the progressive accumulation of triglycerides (TGs) in hepatocytes, which could result from increased free fatty acid (FFA) uptake into the liver, impaired lipid catabolism or enhanced de novo lipogenesis ${ }^{4,5}$.

In recent decades, there have been tremendous advances in understanding the regulatory effect of autophagy on

\footnotetext{
Correspondence: Zhiguo Zhang (zhangzhiguo@outlook.com)

${ }^{1}$ Department of Endocrinology and Metabolism, Shanghai Clinical Center for Endocrine and Metabolic Diseases, Shanghai Institute of Endocrine and Metabolic Diseases, Ruijin Hospital, China National Research Center for Metabolic Diseases, Shanghai Jiao Tong University School of Medicine, 200025 Shanghai, China

These authors contributed equally: Lei Tong, Long Wang, Shuangshuang Yao Edited by G.M Fimia
}

hepatic lipid metabolism. Autophagy is an evolutionarily conserved physiological process that represents a system of bulk protein degradation aimed at the removal and breakdown of cellular components (organelles and proteins) during starvation, thereby redistributing nutrients to maintain cellular energetic balance ${ }^{6}$. It also plays a critical role in eliminating damaged proteins and organelles ${ }^{7}$. Deficiencies in autophagy flux are closely related to the development of hepatic steatosis. Autophagy is supposed to break down intracellular lipids in hepatocytes through a lysosomal degradation pathway and therefore may regulate the development of hepatic steatosis ${ }^{5,8,9}$.

Peroxisome proliferator-activated receptor (PPAR) agonists are well established in therapeutic areas related to lipid and glucose metabolism, such as T2DM, obesity and dyslipidaemia ${ }^{10-12}$. PPAR $\alpha$ is one of the most abundantly expressed nuclear receptors in the $\operatorname{liver}^{12,13}$. PPAR $\alpha$ and its agonists have hepatoprotective effects in rodent models of NAFLD/NASH. However, fibrates and other available PPAR $\alpha$ agonists have shown no beneficial effects on steatosis in human studies ${ }^{14}$. PPAR $\alpha$ expression is low in the human liver relative to the rodent liver, and

\section{(c) The Author(s) 2019}

(c) (i) Open Access This article is licensed under a Creative Commons Attribution 4.0 International License, which permits use, sharing, adaptation, distribution and reproduction in any medium or format, as long as you give appropriate credit to the original author(s) and the source, provide a link to the Creative Commons license, and indicate if changes were made. The images or other third party material in this article are included in the article's Creative Commons license, unless indicated otherwise in a credit line to the material. If material is not included in the article's Creative Commons license and your intended use is not permitted by statutory regulation or exceeds the permitted use, you will need to obtain permission directly from the copyright holder. To view a copy of this license, visit http://creativecommons.org/licenses/by/4.0/. 
this expression level progressively decreases as NASH progresses in humans, which may explain the contradictory results of early PPAR $\alpha$ agonists in randomised clinical trials ${ }^{13,15,16}$. PPAR $\delta$ is ubiquitously expressed and has been implicated in lipid metabolism and energy homoeostasis in various organs, including the liver ${ }^{16}$. Moreover, in recent clinical studies that included overweight patients with mixed dyslipidaemia, there was a reduction in hepatic fat content upon treatment with PPAR $\delta$ agonists ${ }^{17,18}$. However, the exact mechanism by which PPAR $\delta$ attenuates NAFLD remains vague.

To gain insight into the association between PPAR $\delta$ and NAFLD, we examined whether PPAR $\delta$ works against the pathogenesis of NAFLD both in vivo and in vitro. We studied the effects of adenovirus-mediated overexpression and agonist induction of PPAR $\delta$. We demonstrate that autophagy is associated with PPAR $\delta$-induced hepatic fat clearance in vivo by using two rodent models, the $\mathrm{db} / \mathrm{db}$ mouse and the high fat diet-fed mouse, which have been shown previously to mimic human hepatic steatosis. We also show that PPAR $\delta$ activation-induced fatty acid oxidation (FAO) mediated by the autophagy-lysosomal pathway is the central mechanism for improving NAFLD.

\section{Results}

\section{Downregulation of PPAR and autophagy in the liver of} obese mice and ageing mice

The most prominent characteristic of NAFLD is abnormal lipid accumulation in the liver. We selected several models of murine obesity, including both dietary (high fat diet) and genetic (ob/ob and $\mathrm{db} / \mathrm{db}$ ) models. The expression of lipogenic proteins, including fatty acid synthase (FAS), carbohydrate-responsive element binding protein (ChREBP) and stearoyl-CoA desaturase 1 (SCD1), was upregulated in model mice compared to control mice (Fig. 1a-c), which is consistent with increased lipid aggregation in the liver of obese mice ${ }^{19}$. PPAR $\delta$ expression was significantly lower in obese mice than in the respective control mice (Fig. 1a-c). Autophagy proteins showed a significant decrease in obsess mice compared with lean control mice, supported by the downregulation of Atg7, Atg5, Beclin1 and LC3-II (Fig. 1a-c). Normal and pathological ageing is often associated with a reduced autophagic potential. The expression of lipogenic genes was increased in older mice compared to younger mice, and that of autophagic proteins, including Atg7, Atg5, Beclin1 and LC3-II, was significantly decreased. Moreover, PPAR $\delta$ protein levels were reduced in the ageing mice (Fig. 1d). Altogether, the above results suggest that PPAR $\delta$ might have some relationship with autophagy.

\section{PPARס ligand activation upregulates autophagy in primary hepatic cells}

To explore the effect of PPAR $\delta$ on autophagy, we performed an in vitro study with primary mouse hepatocytes
(PMH). Treatment with the PPAR $\delta$ agonist GW501516 increased LC3-II in PMH in a time- and dose-dependent manner (Fig. 2a upper and lower panels). We then treated $\mathrm{PMH}$ with two different PPAR $\delta$ ligands, which resulted in a marked increase in autophagic proteins, including LC3II, Atg5 and Beclin1 and the downregulation of p62 (Fig. 2b).

We next used fluorescence microscopy to observe the effect of PPAR $\delta$ agonists on morphological changes associated with autophagy. Adenovirus carrying the mRFP-GFP-LC3 reporter was used to determine autophagy flux. In this reporter system, mRFP and GFP are used to label and track LC3. While GFP is sensitive to the acidic conditions in autolysosomes, we can detect RFP signals only after the fusion of autophagosomes with lysosomes. The overlay of green and red fluorescence creates yellow dots, and an increase in red spots indicates greater maturation of autolysosomes ${ }^{20,21}$. We observed that PPAR $\delta$ agonists increased both autophagosome (yellow dots) and autolysosome (remaining red dots) formation in merged images (Fig. 2c). Moreover, PPAR $\delta$ agonist-treated PMH were evaluated by electron microscopy (EM). Hepatocytes treated with GW0742 had a significantly increased number of autophagic vacuoles compared with those treated with vehicle control (Fig. 2d). Collectively, these results demonstrate strong proautophagic actions upon PPAR $\delta$ induction.

\section{PPARס ligand increases hepatic lipolysis and overall oxidative metabolism in vivo}

We conducted some preliminary experiments and generated four sets of mouse models. First, we treated high fat diet (HFD)-fed mice and $\mathrm{db} / \mathrm{db}$ mice fed a normal chow diet with the PPAR $\delta$ agonist GW501516. After 4 weeks of GW501516 treatment, fat deposition in liver tissue was significantly improved. Serum TG, ALT and AST levels were decreased, and the mRNA and protein levels of autophagy markers in liver tissue were significantly enhanced (Supplementary Figures S1 and S3). Next, we injected adeno-associated virus (AAV) containing PPAR $\delta$ or GFP (as a control) through the tail vein into C57BL/6 mice that had been fed a HFD and into $\mathrm{db} / \mathrm{db}$ mice. For 4 weeks after AAV injection, C57BL/6 mice continued to be fed a HFD, and $\mathrm{db} / \mathrm{db}$ mice were fed a normal chow diet. Both HFD-fed and db/db mice showed significant improvements in indicators, including serum TG, ALT and AST levels and hepatic TG content. Moreover, autophagy and genes related to FAO were also markedly enhanced compared to control mice (Supplementary Figures S2 and S4).

Chloroquine (CQ) is a well-known pharmacological inhibitor of autophagic and lysosomal function used in vivo $^{22}$ To understand the role of autophagy in PPAR $\delta$ activation-induced lipid turnover in vivo, we 

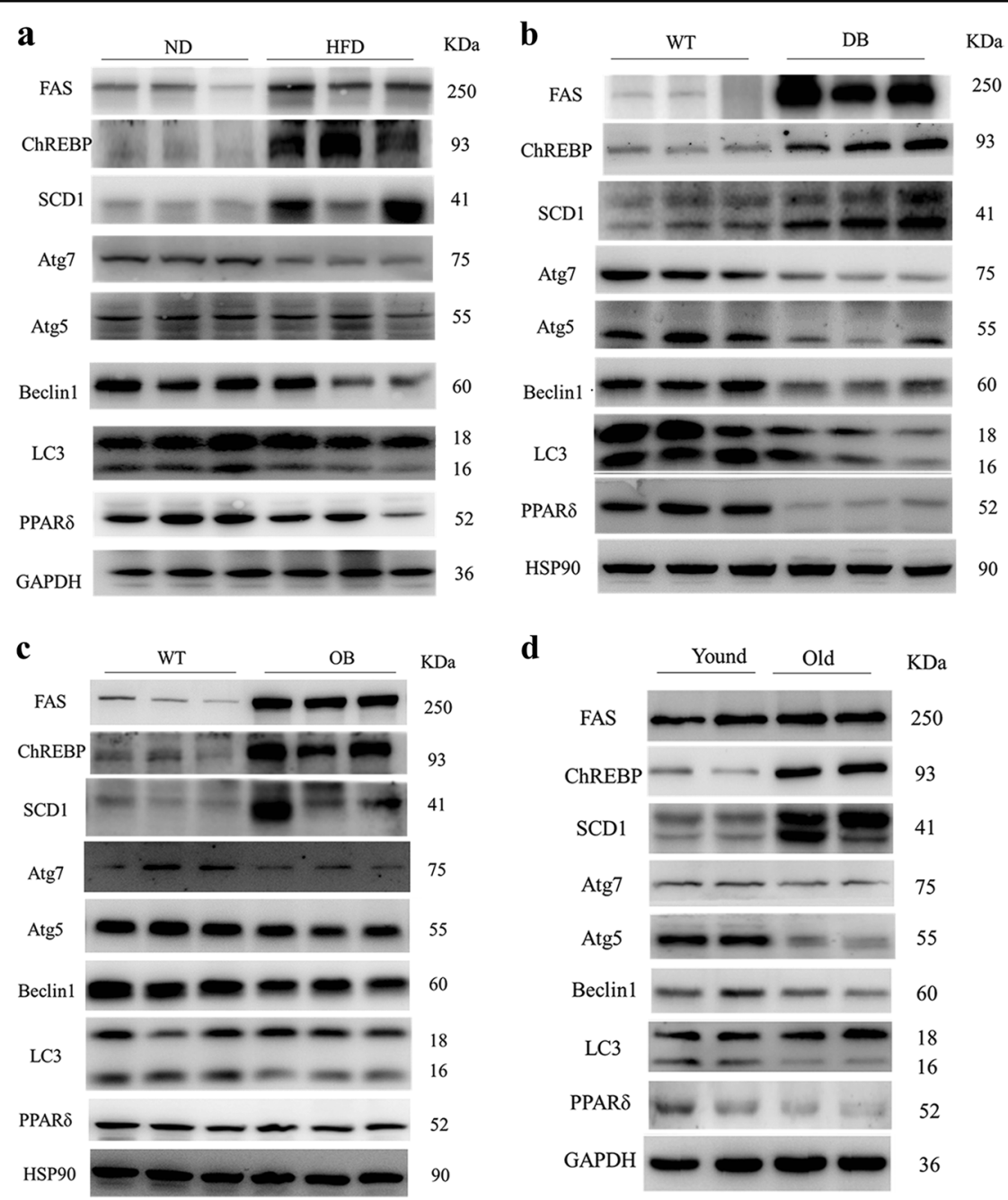

d

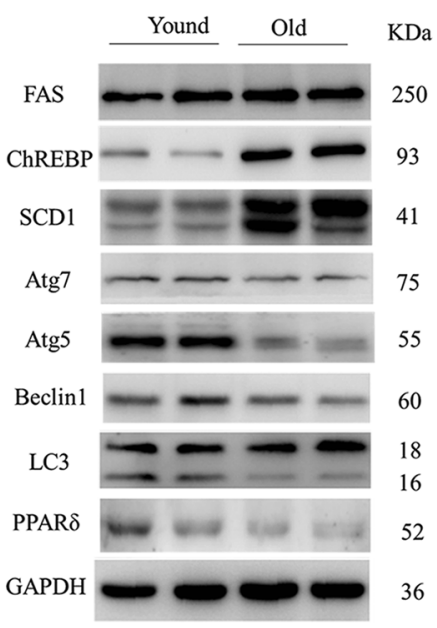

Fig. 1 Reduction in PPAR $\delta$ and autophagy markers in the liver of obese mice. Hepatic protein levels of PPAR $\delta$ and autophagy markers decreased, accompanied by an increase in genes involved in the de novo lipogenesis pathway, in different obese mouse models: a C57BL/6 mice ( $n=6$ per group), $\mathbf{b} \mathrm{db} / \mathrm{db}$ mice ( $n=6$ per group) and $\mathbf{c}$ ob/ob mice ( $n=6$ per group). $\mathbf{d}$ Effects of ageing on lipid metabolism and autophagy as shown by western blot ( $n=6$ per group)

co-administered an autophagy inhibitor to PPAR $\delta$ agonist-treated genetic obesity models. Obese $\mathrm{db} / \mathrm{db}$ mice were separated into four groups, each with the same body weight distribution. The four groups were continuously fed vehicle, GW501516 (oral gavage of $10 \mathrm{mg} / \mathrm{kg}$ b.w. for 7 days) ${ }^{23}$, CQ (IP administration of $30 \mathrm{mg} / \mathrm{kg} \mathrm{b.w}$. for the last 3 days) or GW501516 plus CQ.

After 7 days of treatment, serum ALT and AST levels, as well as serum TGs, were reduced by GW501516. However, in contrast to untreated mice, the GW501516mediated protective effect on liver was abrogated by $C Q$ administration (Fig. 3a-c). Haematoxylin and eosin (H\&E) staining (Fig. 3d) and biochemical analysis of hepatic TG content (Fig. 3e) showed that the steatosis improved by GW501516 treatment was also abolished by CQ administration. Thus, we found that even relatively short-term agonist treatment leads to a vast reduction in lipid accumulation in the liver.

We next sought to explore whether PPAR $\delta$ activation regulates autophagy flux in the liver. The autophagyrelated protein LC3-II was upregulated, and the expression of p62 (an autophagy substrate degraded though autophagy) was reduced in GW501516-treated db/db mice, but this effect disappeared after CQ administration (Fig. 3f). We also performed in vitro study with $\mathrm{PMH}$, PPAR $\delta$ agonist GW501516 or GW0742 treatment also increased LC3-II in PMH (Supplementary Figure S5).

Autophagy is a catabolic process by which cells supply amino acids and FFAs from self-digested organelles and lipids as an alternative energy source for survival. Therefore, the upregulation of autophagy would eventually be associated with the activation of mitochondrial 

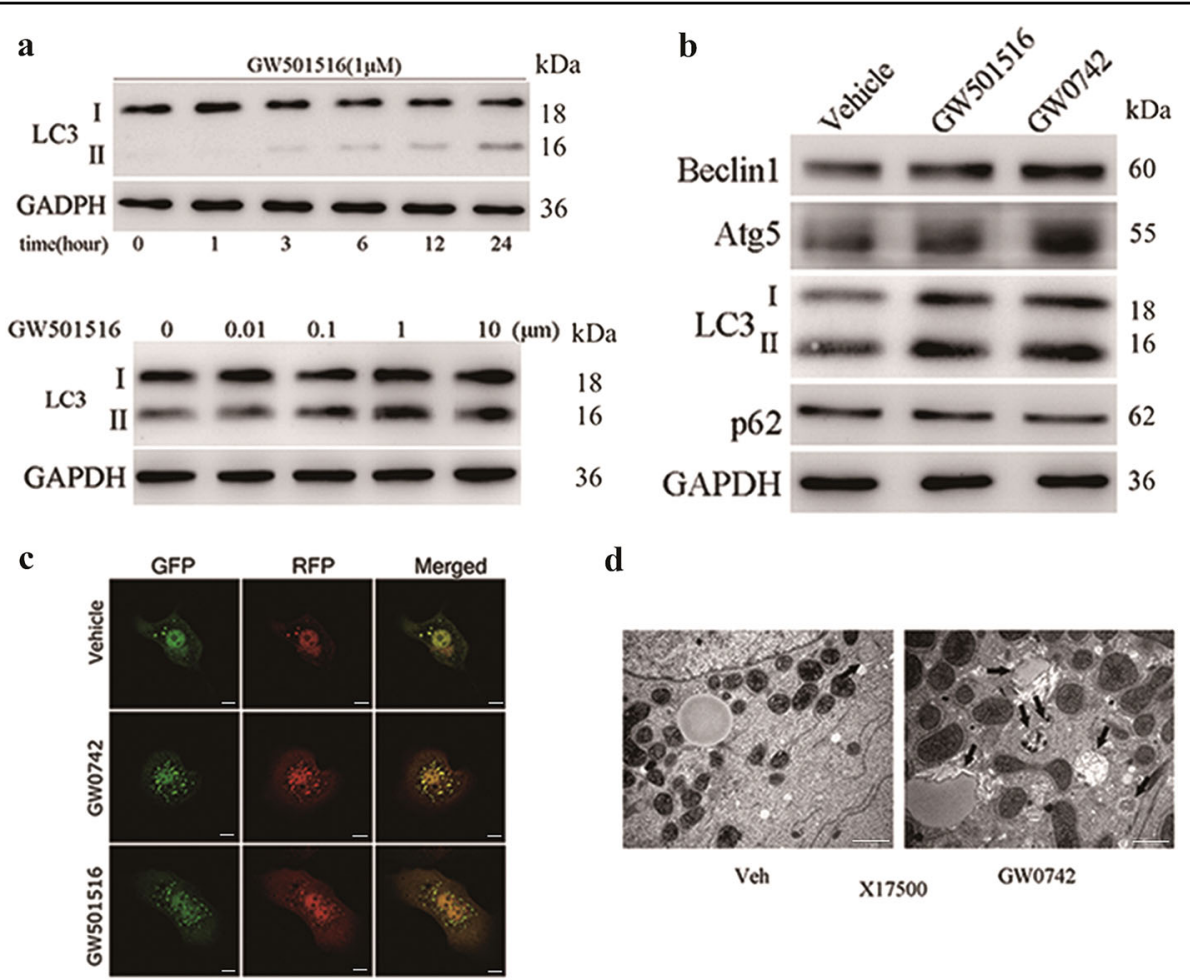

d

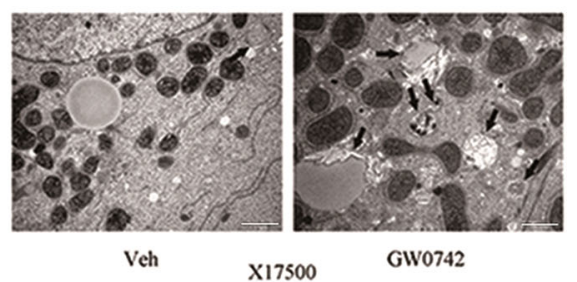

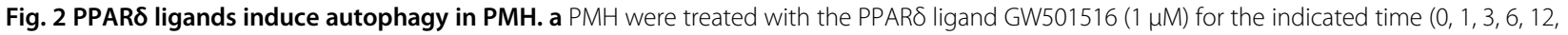
or $24 \mathrm{~h}$ ) or at different concentrations $(0.01,0.1,1$ or $10 \mu \mathrm{M})$ for $24 \mathrm{~h}$, and LC3-II was examined by western blot. b Western blot analysis of autophagy markers after hepatocytes were treated with a PPAR ligand (1 $\mu \mathrm{M}$ GW0742 or GW501516) for 24 h. c Ad-GFP-LC3-infected hepatocytes were treated with GW0742 or GW501516 (both at $1 \mu \mathrm{M}$ ) for $12 \mathrm{~h}$ and examined by fluorescence microscopy. Scale bar: $10 \mu \mathrm{m}$. d Representative EM images of PMH treated with GW0742 for $24 \mathrm{~h}$. Arrows indicate autophagosomes. Scale bar: $1 \mu \mathrm{m}$. Three independent experiments were performed

$\beta$-oxidation ${ }^{21,24}$. $\beta$-Hydroxybutyrate $(\beta-\mathrm{OHB})$ is an end product of hepatic FAO. We measured serum $\beta-\mathrm{OHB}$ levels and assessed the mRNA expression of fatty acid $\beta$ oxidation-related genes by real-time PCR. GW501516 treatment increased serum $\beta-\mathrm{OHB}$ levels and the mRNA expression levels of CPT $1 \alpha$, CPT $1 \beta$, mtTFA, PPARA, COX4b and PDK4. However, the GW501516-mediated increases in $\beta-O H B$ and FAO were abrogated by $C Q$ administration (Fig. 3g, h-m), suggesting that the induction of fatty acid $\beta$-oxidation by GW501516 is dependent upon the autophagy-lysosomal pathway.

The autophagy-lysosomal pathway is essential for the stimulation of $\beta$-oxidation by conditional liver PPAR $\delta$ overexpression in vivo

To establish a direct link between PPAR $\delta$-induced autophagy and hepatic fatty acid $\beta$-oxidation, $\mathrm{db} / \mathrm{db}$ mice with conditional liver PPAR $\delta$ overexpression were treated with or without $C Q$. The four groups received injections of AAV-GFP $\left(1 \times 10^{\wedge} 11 \mathrm{vg} / \mathrm{ml}\right.$ for 4 weeks $)$, AAV-PPAR $\delta$ $\left(1 \times 10^{\wedge} 11 \mathrm{vg} / \mathrm{ml}\right)$, AAV-GFP plus CQ (IP administration of $30 \mathrm{mg} / \mathrm{kg}$ for the last 3 days), or AAV-PPAR $\delta$ plus CQ.

Similar to the results for obese mice fed GW501516 plus CQ, the reduction in serum ALT and AST levels in the AAV-PPAR $\delta$ group vanished completely after CQ administration (Fig. 4a, b). Furthermore, H\&E-stained liver sections showed that hepatic steatosis was greatly improved, and PPAR $\delta$ overexpression significantly decreased hepatic TG levels in a quantitative assay. However, these beneficial effects were also abolished by CQ administration (Fig. 4c, d).

The protein levels of autophagy markers, such as LC3, Atg5, Atg7 and Beclin1, increased markedly in the AAVPPAR $\delta$ group, and p62 was significantly downregulated. However, the effects disappeared totally after CQ coadministration (Fig. 4e). Conditional PPAR $\delta$ overexpression increased ketogenesis and the expression of genes related to $\mathrm{FAO}$, and these changes were abrogated in the presence of CQ (Fig. 4f, g-l). These data suggest that PPAR $\delta$-induced $\beta$-oxidation occurs specifically through the activation of autophagy in vivo.

\section{Autophagy mediates PPARס-induced oxidative metabolism in hepatic cells}

To determine whether autophagy induced by PPAR $\delta$ is directly involved in reducing intracellular lipid content, we assessed the effects of lysosomal inhibition on the reduction in intracellular lipids by PPAR $\delta$ in PMH. We 


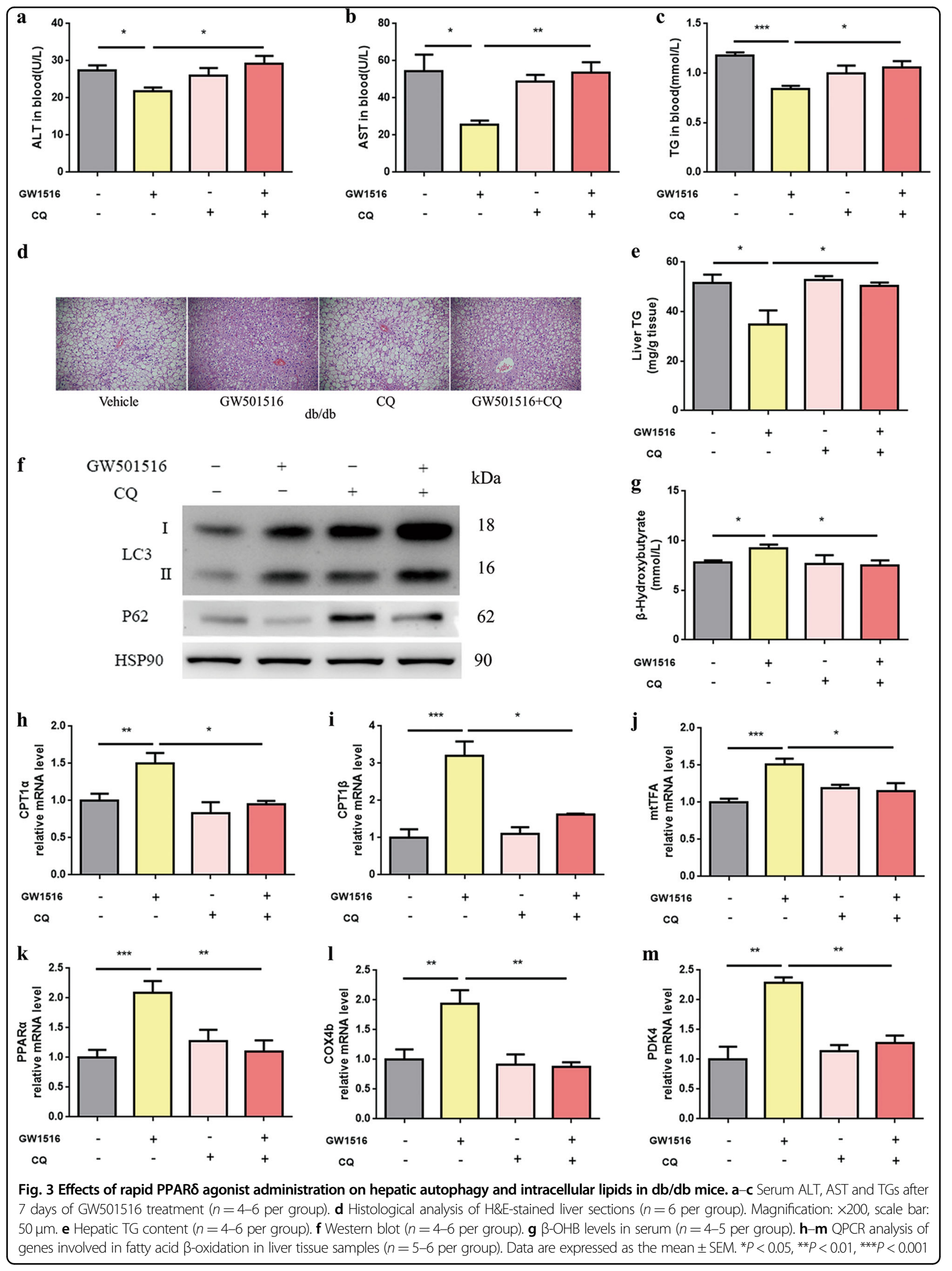




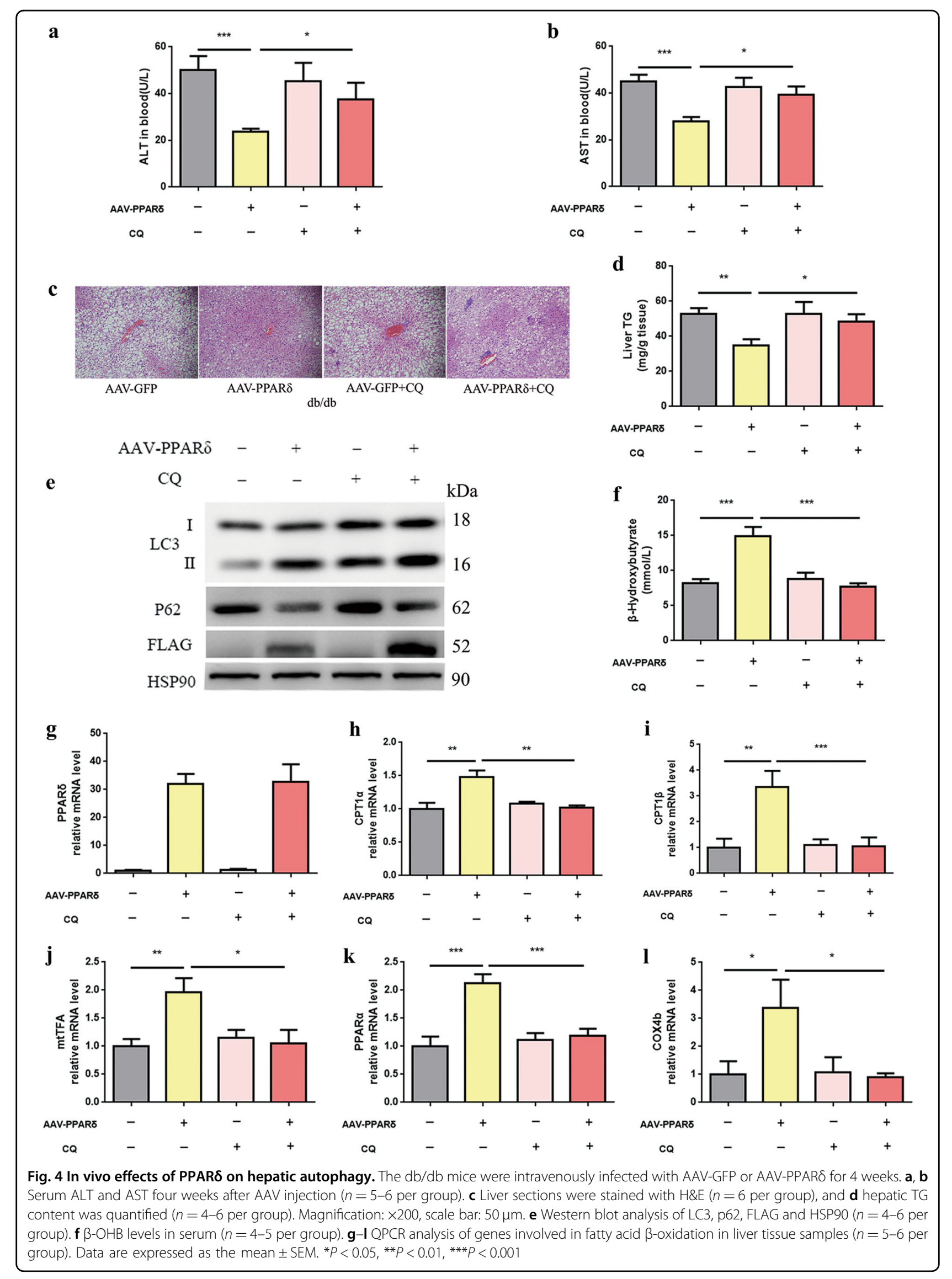




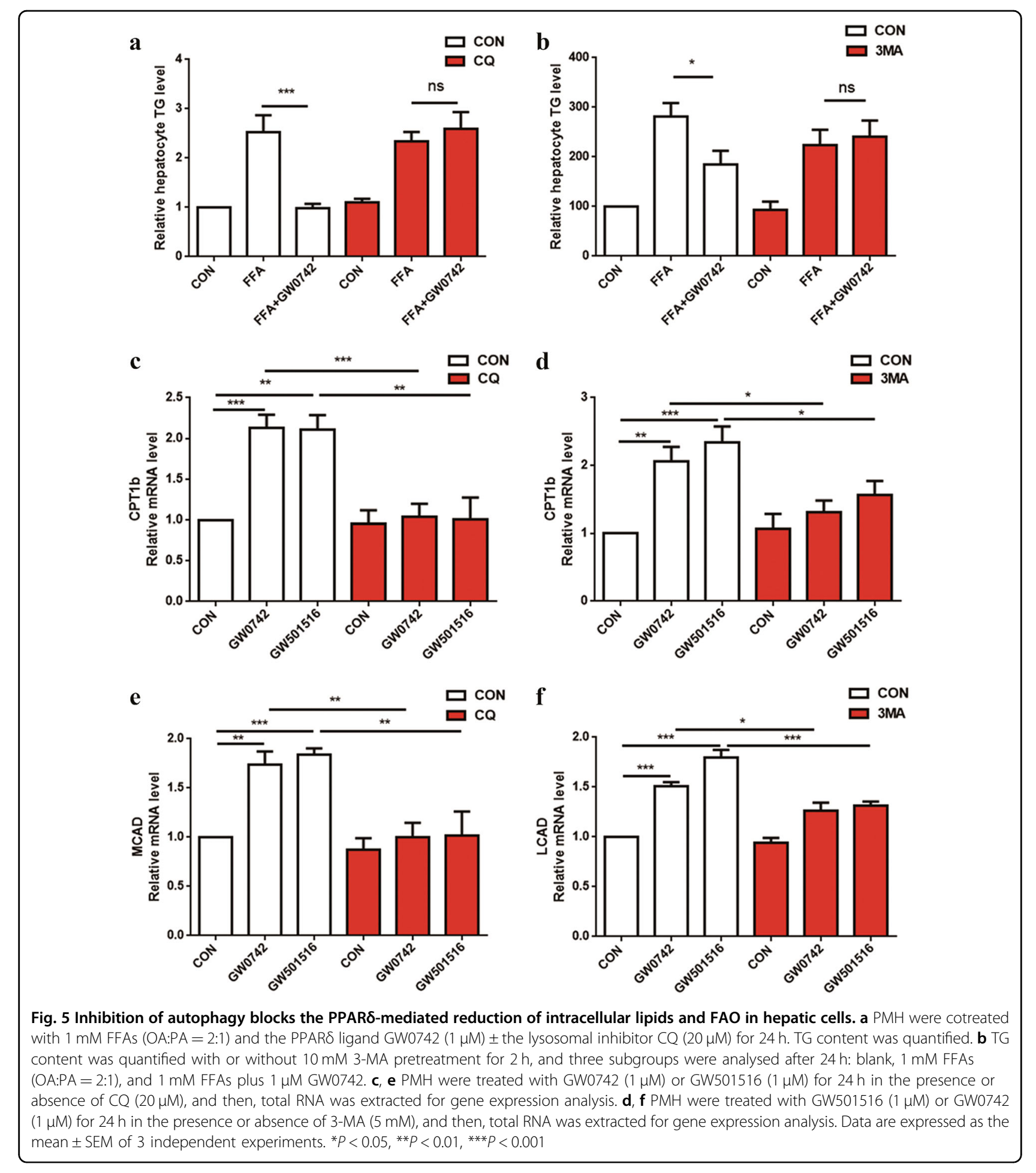

used the PPAR $\delta$ agonists GW0742 and GW501516 and the autophagy inhibitors $\mathrm{CQ}$ and 3-methyladenine (3-MA) in our study. CQ can interfere with the lysosome $\mathrm{pH}$ and thus suppress lysosome-mediated degradation, which would lead to the inhibition of autophagy ${ }^{25}$. 3-MA, a widely used autophagy inhibitor, inhibits the class III PI-3 kinase required for autophagy induction, which has been shown to dampen autophagy ${ }^{20,22}$.

As shown in Fig. 5a, b, treatment with $1 \mathrm{mM}$ FFAs significantly increased cellular TG levels, and GW0742 significantly weakened the FFA-induced lipid accumulation in $\mathrm{PMH}$. However, this improvement in 
lipid accumulation upon PPAR $\delta$ activation in hepatocytes was completely abolished by pretreatment with CQ or 3-MA (Fig. 5a, b). CQ and 3-MA also abolished the increased expression of genes related to fatty acid $\beta$-oxidation, which suggested that autophagy is essential for PPAR $\delta$-induced fat oxidation and TG clearance (Fig. $5 \mathrm{c}-\mathrm{f}$ ). The above data demonstrate the involvement of the autophagy-lysosomal pathway in the reduction in hepatic intracellular lipids by PPAR $\delta$.

\section{Autophagy inhibition impairs the PPAR $\delta$-induced effects on intracellular lipid content and oxidative metabolism in vitro}

We used ATG5 siRNA to block autophagosome formation. The data showed that ATG5-targeted RNA interference efficiently abrogated ATG5 gene and protein expression (Fig. 6a, b). ATG5 knockdown (KD) impaired PPAR $\delta$ agonist-induced effects. Additionally, ATG5 KD abolished GW501516-induced autophagy by decreasing the expression of the autophagy-related protein LC3-II and increasing the protein expression p62, an autophagy substrate (Fig. 6b).

GW501516 treatment increased the expression of genes related to FAO, but this effect was blocked by ATG5 KD (Fig. 6e, f). Furthermore, ATG5 KD also abolished PPAR $\delta$ activation-induced $\beta$-OHB production in HepG2 cells (Fig. 6d). These results suggest that autophagy is essential for PPAR $\delta$-induced FAO.

Most importantly, ATG5 siRNA dramatically impaired the downregulation of intracellular lipids by GW501516, strongly suggesting the involvement of autophagy in the PPAR $\delta$-induced reduction of intracellular lipids (Fig. 6c).

\section{PPAR $\delta$ is required to upregulate autophagy in vitro}

The short hairpin RNA (shRNA)-mediated KD of PPAR $\delta$ was used to assess the specific function of PPAR $\delta$ in the regulation of autophagy in vitro. As shown in Fig. 7a, b, PPAR $\delta$-targeted RNA interference efficiently abrogated PPAR $\delta$ gene and protein expression. We found that PPAR $\delta$ KD was sufficient to inhibit autophagy under basal conditions, as reflected by decreased LC3-II levels and increased p62 expression. Furthermore, PPAR $\delta$ KD significantly decreased the phosphorylation of AMP-activated protein kinase (AMPK) and increased p-mammalian target of rapamycin (mTOR) levels (Fig. 7b). PPAR $\delta$ KD also abolished GW501516-induced FAO in HepG2 cells (Fig. 7c).

\section{PPARס facilitates autophagy through AMPK/mTOR pathway}

We measured the phosphorylation levels of AMPK and mTOR levels in vivo, and in all four different mouse models, we found that AMPK phosphorylation was significantly increased and that mTOR phosphorylation was decreased by PPAR $\delta$ overexpression (Fig. $8 \mathrm{a}-\mathrm{d}$ ). Next, we performed an in vitro study with $\mathrm{PMH}$ and treated these cells with two different PPAR $\delta$ ligands, GW501516 and GW0742. The results showed a marked increase in p-AMPK and the downregulation of p-mTOR (Fig. 8e).

A schematic diagram of the induction of PPAR $\delta$ induced FAO mediated by the autophagy-lysosomal pathway is shown in Fig. 8f.

\section{Discussion}

In this study, we have shown that PPAR $\delta$ overexpression or agonist induction prevents hepatic steatosis in obese mice. Our findings are concordant with those of a previous clinical trial, which showed a reduction in liver fat in moderately obese men after two weeks of GW501516 treatment ${ }^{18}$. Here, we also identified and characterised a novel mechanism by which PPAR $\delta$ effectively increases hepatic FAO and autophagic flux. Our study may provide a critical mechanism to narrow the gap between basic research and clinical applications.

Although increased supply of FFAs, hepatic lipogenesis, decreased FAO, and the hepatic secretion of TG-rich, very low-density lipoprotein have all been observed in the development of NAFLD, the primary reason for lipid accumulation in the liver is not yet clear $^{26-28}$. In recent years, there has been intense interest in autophagy, which could be manipulated for the management of NAFLD ${ }^{26,29}$. As reported in 2009 by Rajat Singh et al., the inhibition of autophagy in cultured hepatocytes and the mouse liver increased TG storage in lipid droplets ${ }^{30}$, and autophagyenhancing drugs could alleviate liver steatosis in HFD-fed mice ${ }^{31}$. It should be noted that some common drugs used in the treatment of metabolic disease, such as metformin, mitigate hepatic fat content in a manner involving the autophagy machinery ${ }^{32}$. In particular, we showed that PPAR $\delta$ effectively decreases intracellular TGs and increases autophagic flux in primary hepatic cells and in animals. Activation of the autophagy machinery was demonstrated on several levels, including by examining LC3 turnover in the absence or presence of CQ to measure the induction of autophagy and by using fluorescence microscopy to visualise autophagosome formation via LC3 localisation. Although the experiments were performed in different tissues, other researchers have demonstrated that GW501516 induces autophagic markers in cultured cardiac cells and in the heart of HFD-fed mice $^{33,34}$.

In fasting conditions, the body can hydrolyse TG to supply FFAs that meet the energy needs ${ }^{35}$. Hepatic FAO is enhanced in the fasted state $^{36}$ and an alternative energy source with respect to energy deprivation is provided by the breakdown of cellular components by autophagy ${ }^{35,37}$. Defective autophagy is involved in $\mathrm{NAFLD}^{30}$, and the induction of autophagy can correct the 
a

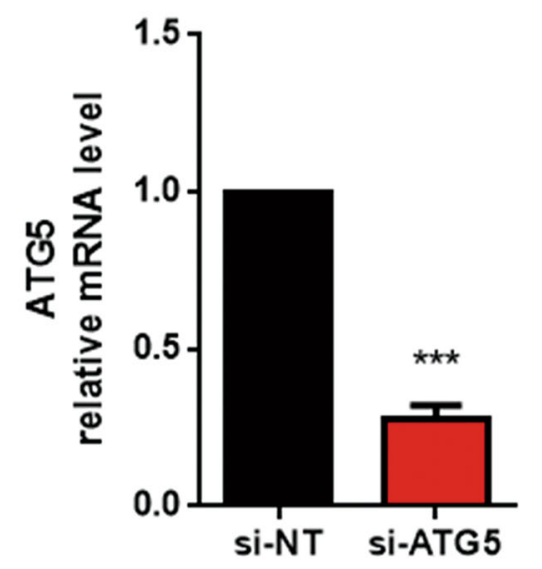

c

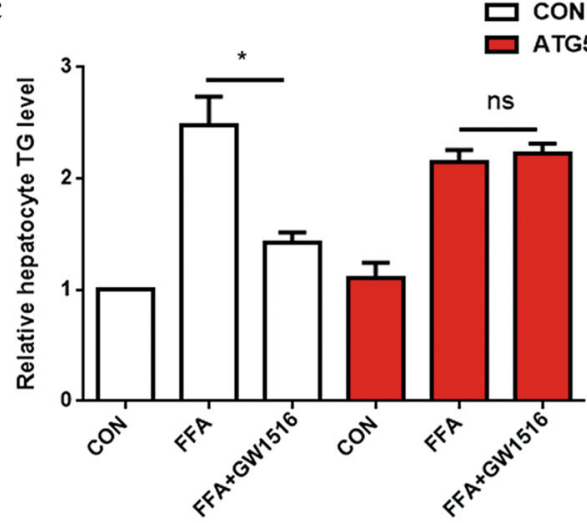

e

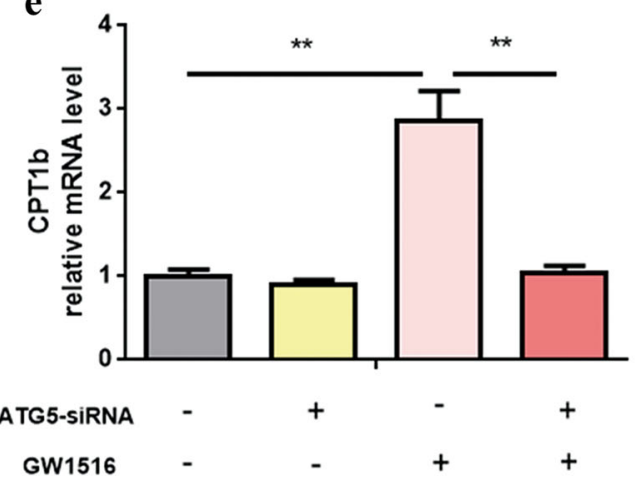

b

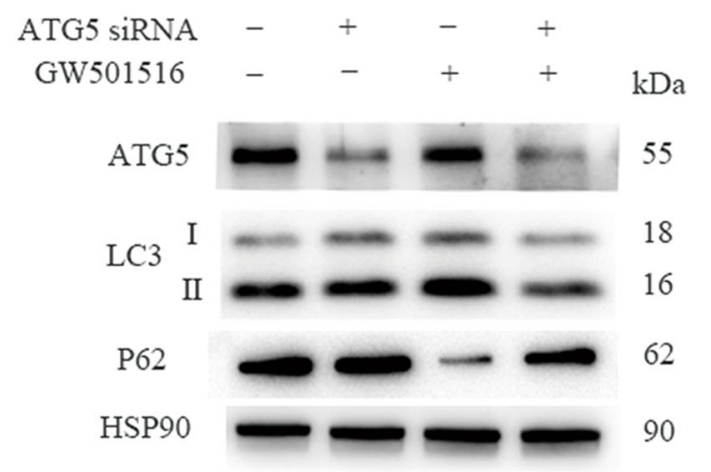

d

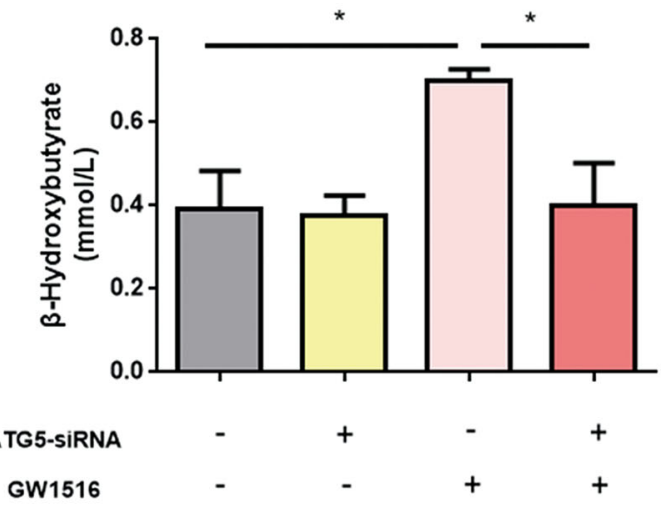

f

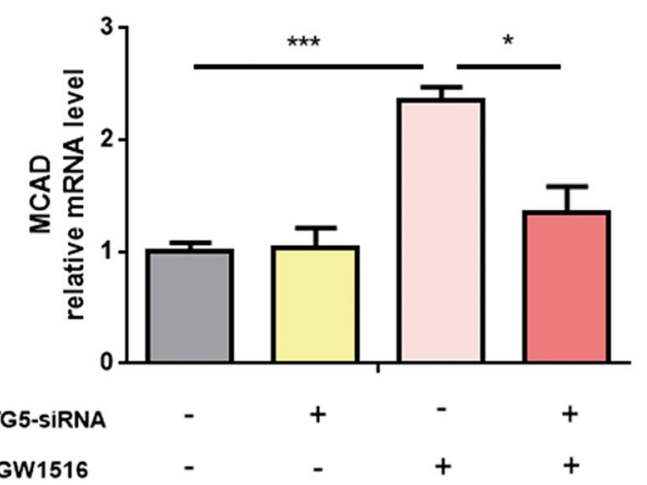

Fig. 6 Role of autophagy in the increased oxidative metabolism and reduced intracellular lipids in HepG2 cells. a HepG2 cells were transiently transfected with $60 \mathrm{nmol} / \mathrm{L}$ ATG5 siRNA or negative control siRNA for $48 \mathrm{~h}$, and then, QPCR analysis of the ATG5 gene was assessed to indicate knockout efficiency. $\mathbf{b}$ HepG2 cells were first treated with $60 \mathrm{nmol} / \mathrm{L}$ ATG5 siRNA for $24 \mathrm{~h}$ and then with $1 \mathrm{mM} F F A s(O A: P A=2: 1) \pm$ the

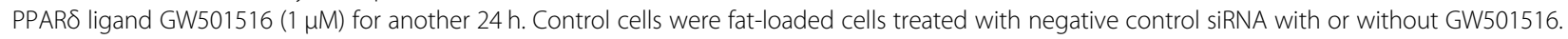
Western blot analysis of autophagy markers. c, $\mathbf{d}$ TG content and $\beta$-OHB measurement in culture media from HepG2 cells \pm ATG5 KD (60 nmol/L) for $24 \mathrm{~h}$, followed by a 24-hour treatment with or without $1 \mathrm{mM}$ lipid mixture containing OA and PA at a 2:1 ratio \pm GW501516 (1 $\mu$ M). Control cells were fat-loaded cells treated with negative control siRNA with or without GW501516. e, f QPCR analysis of genes involved in fatty acid $\beta$-oxidation. Data are expressed as the mean \pm SEM of 3 independent experiments. ${ }^{*} P<0.05,{ }^{* *} P<0.01,{ }^{* *} P<0.001$

over-accumulation of lipids in the $\operatorname{liver}^{38}$. We used pharmacological (CQ and 3-MA) and genetic (ATG5 siRNA) methods to modulate autophagy and lysosomes to demonstrate that the autophagy-lysosomal pathway is essential for the reduction of intracellular fat and the stimulation of oxidative metabolism by PPAR $\delta$. 


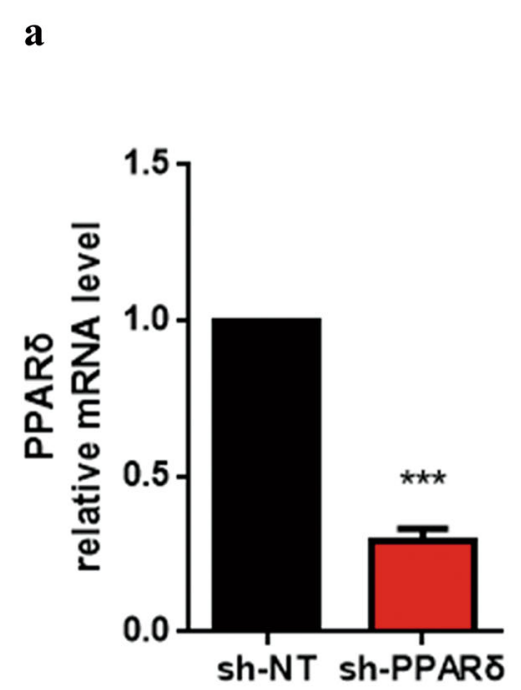

c

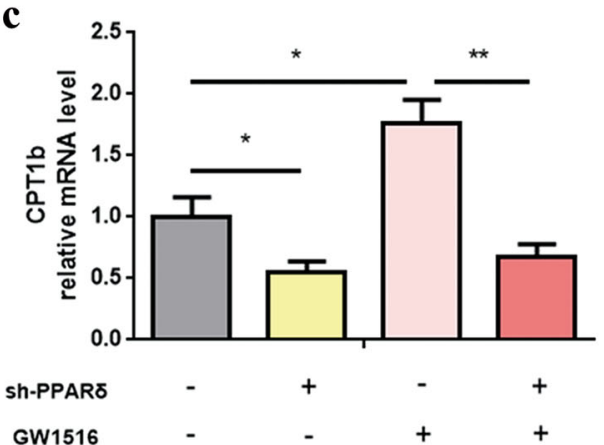

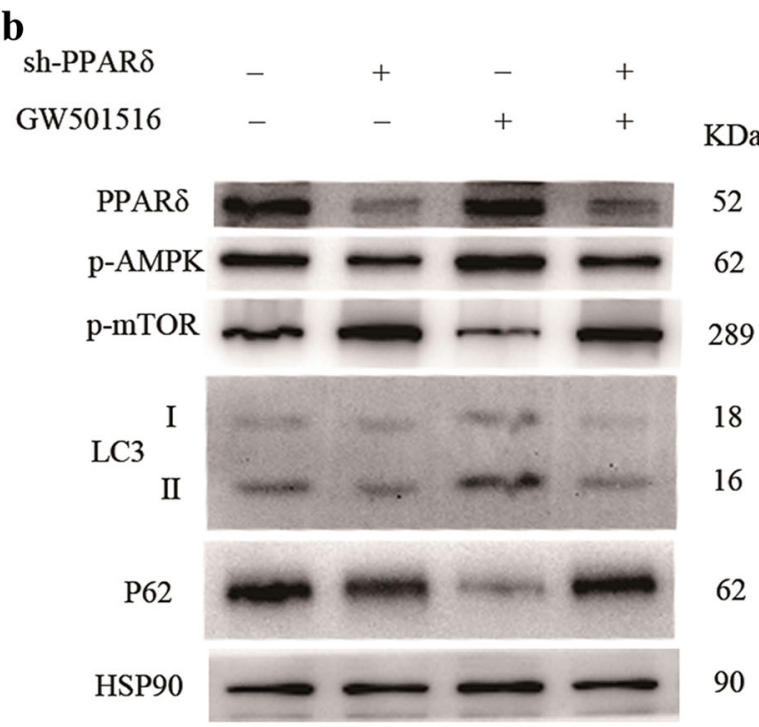

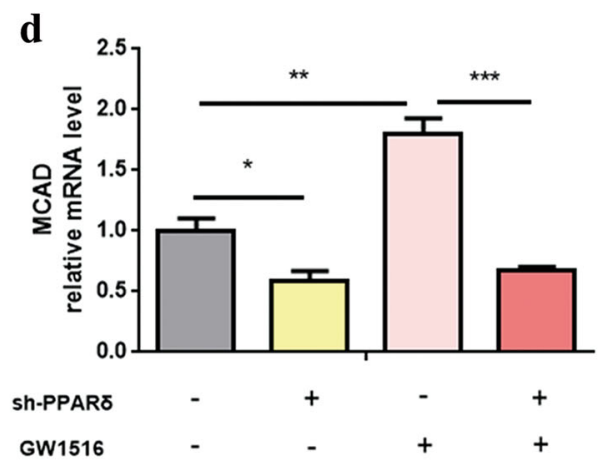

Fig. 7 PPARס is required to upregulate autophagy in vitro. a HepG2 cells were infected with lentivirus-mediated control shRNA or PPARס shRNA at a multiplicity of infection of 10 in 6-well plates for $72 \mathrm{~h}$; then, QPCR analysis of PPAR gene expression was performed to assess the knockout

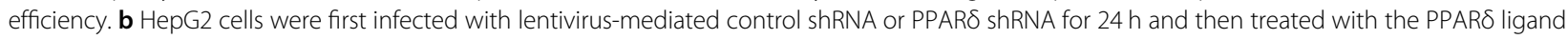
GW501516 (1 MM) for another $72 \mathrm{~h}$. Control cells were cells treated with negative control shRNA with or without GW501516. Protein expression levels were determined by western blot. $\mathbf{c}$, $\mathbf{d}$ QPCR analysis of genes involved in fatty acid $\beta$-oxidation. Data are expressed as the mean \pm SEM of 3 independent experiments. ${ }^{*} P<0.05,{ }^{* *} P<0.01,{ }^{* *} P<0.001$

We treated primary hepatic cells with PPAR $\delta$ in the presence of CQ, 3-MA or ATG5 siRNA to establish a direct link between PPAR $\delta$-induced autophagy and hepatic fatty acid $\beta$-oxidation. It has been reported that PPAR $\delta$ can improve hepatic steatosis by activating fatty acid $\beta$-oxidation in different diet-induced models of steatohepatitis (NASH, obesity and insulin resistance) $)^{39-41}$. Our study clearly demonstrates the importance of autophagy and lysosomal degradation in promoting fatty acid $\beta$-oxidation, which might be the key pathway through which PPAR $\delta$ maintains intrahepatic lipid homoeostasis.

Our results also showed increased AMPK phosphorylation and mTOR inhibition in vivo and in vitro upon PPAR $\delta$ induction. Autophagy can be promoted by the energy sensor AMP-activated protein kinase (AMPK) and be inhibited by the mammalian target of rapamycin $(\mathrm{mTOR})^{42}$. Although further investigations need to be done, it is possible that the underlying mechanism of PPAR $\delta$ activated autophagy formation at least partly involves the AMPK/mTOR pathway.

Another reason why PPAR $\delta$ is a promising drug for targeting NAFLD is its expression in different cell types of the liver. PPAR $\delta$ is expressed in hepatocytes, Kupffer cells and stellate cells ${ }^{43}$. In accordance with the beneficial effects of PPAR $\delta$ in improving hepatic steatosis, PPAR $\delta$ could also protect against liver inflammation and fibrosis $^{40,43,44}$.

In summary, we have demonstrated that PPAR $\delta$ has a beneficial effect in attenuating hepatic steatosis by activating autophagy in vivo and in vitro. The mobilisation and hydrolysis of TGs to FFAs through the autophagy-lysosomal pathway induced by PPAR $\delta$ led to increased delivery of FFAs to mitochondria. This, in turn, increased the $\beta$-oxidation of fatty acids. These findings are 


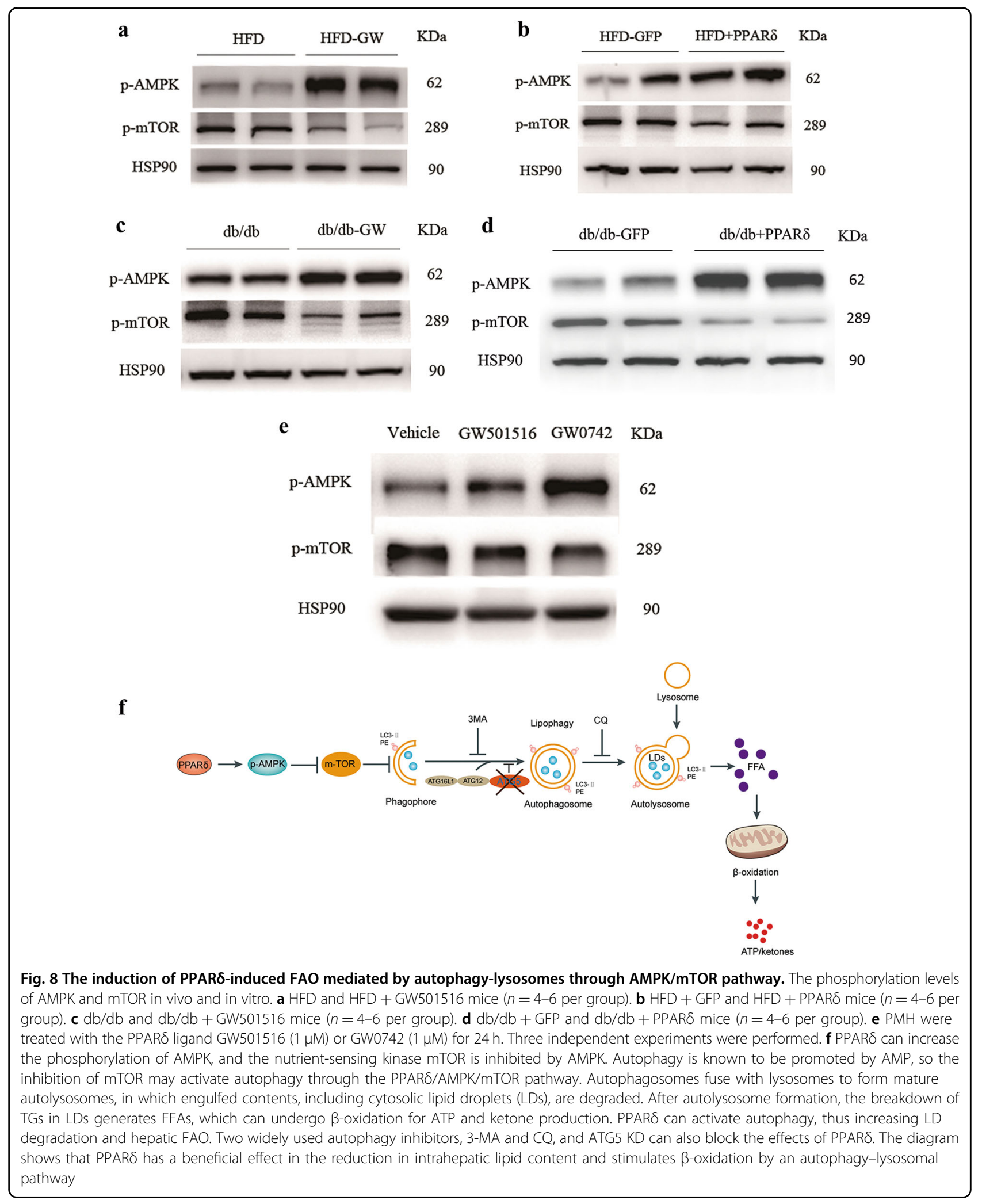


especially noteworthy because it is possible that PPAR $\delta$ activation will be a pharmacological tool for activating autophagy. Understanding the mechanistic basis of PPAR $\delta$ provides further insight into the development of more drugs for the prevention and treatment of NAFLD.

\section{Materials and methods}

\section{Chemicals and antibodies}

GW501516, GW0742 and 3-MA were purchased from Sigma-Aldrich (MO, USA). The antibodies used for western blot included anti-LC3A/B, anti-p62, anti-Atg7, and anti-Beclin1 (Cell Signaling Technology); anti-Atg5 (Novus Biologicals); anti-GAPDH (KangCheng Bio-tech); and anti-PPAR $\delta$ (Santa Cruz Biotechnology). All the other chemicals were from Sigma-Aldrich Corporation unless specifically noted.

\section{Cell culture}

$\mathrm{PMH}$ were fractionated by collagenase digestion from wild-type C57BL/6J mice aged 6-10 weeks. After being filtered and washed with PBS, the hepatocytes were seeded on new plates in attachment media (Hepatocyte Medium, ScienCell) for $24 \mathrm{~h}$ before further experiments. The culture medium was replaced with DMEM (Dulbecco's Modified Eagle's Medium, GIBCO) when the hepatocytes were treated with GW501516 or other chemicals. The cells were always cultured in a $37^{\circ} \mathrm{C}$ incubator with $5 \% \mathrm{CO}_{2}$.

HepG2 cells were maintained at $37^{\circ} \mathrm{C}$ in DMEM (GIBCO) supplemented with $10 \%$ foetal bovine serum.

\section{Animal experiments}

The animal protocol was reviewed and approved by the Animal Care Committee of Shanghai Jiao Tong University School of Medicine. Male C57BL/6J mice were purchased from Shanghai Laboratory Animal Co., Ltd. (SLAC). Obese C57BLKS/J-Leprdb/Leprdb (db/db) male mice and their wild-type littermates were purchased from the Model Animal Research Center of Nanjing University. At 8 weeks of age, the mice were housed at $22 \pm 2{ }^{\circ} \mathrm{C}$ and 55 $\pm 5 \%$ relative humidity with a 12 -hour light/dark cycle. HFD-induced obese mice had free access to high fat chow (D12492, Research Diets, New Brunswick, New Jersey, USA) and drinking water beginning at 8 weeks old. This diet contains $60 \%$ fat, $20 \%$ protein and $20 \%$ carbohydrate by energy.

\section{Tandem mRFP-GFP fluorescence microscopy}

Adenovirus expressing tandem mRFP-GFP-LC3 was purchased from Hanbio Biotechnology Co., Ltd., and was used to monitor autophagic flux in primary hepatocytes. Twenty-four hours after transfection, RFP and GFP were detected in hepatocytes. Then, GW0742, GW501516 or vehicle was added, and $9 \mathrm{~h}$ later, the hepatocytes were washed with PBS and fixed with paraformaldehyde. The samples were sent out to be scanned and photographed by confocal microscopy.

\section{Transmission electron microscopy}

Cells were washed with PBS and then fixed in 2.5\% glutaraldehyde in PBS for $2 \mathrm{~h}$ at $4{ }^{\circ} \mathrm{C}$. After two washes with PBS, the cells were postfixed in $1 \%$ osmium tetroxide in $0.1 \mathrm{M}$ phosphate buffer for $2 \mathrm{~h}$. Samples were dehydrated with increasing concentrations of ethanol and then embedded in epoxy resin. Slices were cut with an ultramicrotome and sent to be photomicrographed under a transmission electron microscope (PHILIP, CM-120, Holland).

\section{Immunoblotting}

Homogenised tissues and cells were lysed in RIPA buffer with protease and phosphatase inhibitors. After separation by SDS-PAGE, the proteins in each sample was transferred to polyvinylidene fluoride membranes (Millipore). The membranes were blocked, incubated with different primary antibodies and then incubated with horseradish peroxidase-conjugated secondary antibodies following the manufacturer's protocol. Protein bands were visualised by chemiluminescence with ECL Prime Western Blotting Detection Reagent (GE Healthcare) and quantified using AlphaView Software (for results, see Supplementary Fig 6).

\section{Quantitative real-time PCR analysis}

After $1 \mu \mathrm{g}$ of total RNA was extracted from each sample with Trizol reagent (Invitrogen) following the manufacturer's instructions, cDNA was prepared using the Reverse Transcription System (Promega). The relative mRNA expression level was determined by quantitative real-time PCR with SYBR Premix (Takara Bio, RR420A) and a Thermo Fisher QS DX Real-time PCR system.

\section{Histological examination}

Liver tissues were fixed in 4\% paraformaldehyde overnight immediately after dissection, embedded in paraffin and sectioned. After H\&E staining, the slices were visualized and imaged by light microscopy at $\times 200$ magnification.

\section{Hepatic and cellular TG measurement}

Mice were sacrificed after an overnight fast (16 h), and liver tissues were preserved in liquid nitrogen. Approximately $50 \mathrm{mg}$ of liver tissue was sliced and homogenised in $1 \mathrm{ml}$ of $5 \%$ NP-40 buffer. Then, the samples were incubated in a water bath at $80-100^{\circ} \mathrm{C}$ for 5 minutes, cooled to room temperature; the heating and cooling process was repeated once. Next, the samples were centrifuged at $13,300 \mathrm{rpm}$ for 2 minutes to remove any 
insoluble material. The TG concentration was measured using a Triglyceride Quantification Kit (Biovison, K622-100). PMH were resuspended in 5\% NP-40 buffer, and TG content was detected as described above.

\section{Autophagy inhibition and intracellular TG measurement in vitro \\ PMH and HepG2 cells were cotreated with $1 \mathrm{mM}$ FFAs $(\mathrm{OA}: \mathrm{PA}=2: 1)$ and PPAR $\delta$ ligand $(1 \mu \mathrm{M}$ GW0742 or GW501516) with or without the autophagy inhibitor CQ $(20 \mu \mathrm{m})$ or 3 -MA $(5 \mathrm{mM})$ for $24 \mathrm{~h}$. TG content was quantified.}

\section{Acute GW501516 and CQ administration and ketogenesis measurements}

GW501516 (10 mg/kg b.w.) was orally gavaged daily for 7 days in male $\mathrm{db} / \mathrm{db}$ mice fed a normal chow diet (NCD), and tissues were collected for western blotting and realtime PCR analysis. To assess the effect of autophagy inhibition on PPAR $\delta$-induced fat oxidation in vivo, CQ was injected IP daily for the last 3 days in male $\mathrm{db} / \mathrm{db}$ mice (30 mg/kg b.w.), and $\beta$-OHB levels were assessed using a commercially available colorimetric kit (Jiancheng Bioengineering Institute, Nanjing).

\section{Liver-restricted PPAR overexpression and CQ administration in vivo}

AAV expressing murine PPAR $\delta$ was constructed by Hanbio Biotechnology Co., Ltd., with the full-length cDNA for PPAR $\delta$ containing a FLAG tag. Overexpression of PPAR $\delta$ was achieved by AAV-PPAR $\delta(1 \times$ $10^{\wedge} 11 \mathrm{vg} / \mathrm{ml}$ ) injection into $\mathrm{db} / \mathrm{db}$ mice through the tail vein, and AAV-GFP was used as a control. The $\mathrm{db} / \mathrm{db}$ mice continued to be fed a NCD for another fourweeks after the AAV injection, and CQ (30 mg/ kg b.w. IP for the last three days) was co-administered with AAV injections. Tissues were collected for western blotting, real-time PCR analysis and $\beta-\mathrm{OHB}$ measurements.

\section{Targeted KD of ATG5 by siRNA}

ATG5 KD was carried out using Stealth siRNA duplex oligoribonucleotides targeting human ATG5 (Invitrogen). After 24h, HepG2 cells were treated with the PPAR $\delta$ ligand GW501516 $(1 \mu \mathrm{M})$ and $1 \mathrm{mM}$ FFAs (OA:PA = 2:1). Measurements of intracellular levels were assessed after $24 \mathrm{~h}$ of cotreatment. The siRNA oligonucleotide duplex (5-GGU UUG GAC GAA UUC CAA CUU GUU U-3) to human ATG5 was synthesised by Invitrogen, Carlsbad, CA (Stealth Select RNAi). Stealth RNAi negative control with low GC content was ordered directly from Invitrogen (catalogue number 12935200). Transient transfection of synthetic siRNA was achieved using Lipofectamine 2000 transfection reagent (Invitrogen). The transfection efficiency of each duplex siRNA (>90\%) was confirmed by using BLOCK-iT ${ }^{\mathrm{TM}}$ Alexa Fluor ${ }^{\mathrm{TM}}$ Red Fluorescent Control (Invitrogen, catalogue number 14750100) according to the manufacturer's instructions.

\section{Lentivirus transduction}

The lentivirus containing sense-loop-antisense shRNA targeting human PPAR $\delta$ was purchased from ShanghaiXitubio Corporation. The shRNA sequences were as follows: PPAR $\delta$, GATCAAGAAGACCGAAACCGA; and negative control, GCGCGATAGCGCTAATAATTT. PPAR $\delta$ shRNA was subcloned into the pLKO5-GFP lentiviral vector to obtain the pLKO5-GFP-sh-PPAR $\delta$ construct. Then, HepG2 cells were infected with pLKO5-GFP-sh-PPAR $\delta$ or negative lentiviral vectors at a multiplicity of infection of 10 . The efficiency of infection at $72 \mathrm{~h}$, which was determined by the number of GFPpositive cells, exceeded $90 \%$ for all constructs.

\section{Statistical analysis}

Statistical analyses were performed with SPSS version 23.0 (SPSS Inc., Chicago, IL, USA) and GraphPad Prism version 6.0 (GraphPad Software Inc., San Diego, CA, USA). Continuous data are presented as the mean \pm standard error of the mean (SEM) unless otherwise specified. The Kolmogorov-Smirnov test (using SPSS) or Bartlett's test (using GraphPad Prism) was performed to verify the normality of each variable, and the Brown-Forsythe test was performed to test the equality of group variances. For the comparison of two groups, the unpaired Student's $t$-test was used if the variances were equal; otherwise, the $t$-test was performed. For the comparison of three or more groups, one-way analysis of variance (ANOVA) with Tukey's post hoc adjustment was performed. A two-tailed $P$ value less than 0.05 was considered statistically significant.

All the data are presented as the mean \pm SEM. Statistical differences were set as ${ }^{*} p<0.05,{ }^{* *} p<0.01$ and ${ }^{* * * *} p<$ 0.001 .

\footnotetext{
Acknowledgements

This study was supported by the National Natural Science Foundation of China (Nos. 81570719, 81670797 81471074, 81770863, and 81500660) with partial support from the Key Laboratory for Endocrine and Metabolic Diseases, Chinese Ministry of Public Health (No. 1994DP131044), the Ministry of Science \& Technology Innovation Fund and Projects (No. 2011YQ030114), the Basic Important Program (No. 10JC141100), the Sector Funds of the Ministry of Health (No. 201002002), and the National Key New Drug Creation and Manufacturing Program by the Ministry of Science and Technology (No. 2012ZX09303006-001). This study was supported by the Shanghai Municipal Natural Science Foundation (18ZR1433000) the Fund of Shanghai Municipal Education Commission and Shanghai Education Development Foundation (No. 14SG17).
}

Conflict of interest

The authors declare that they have no conflict of interest. 


\section{Publisher's note}

Springer Nature remains neutral with regard to jurisdictional claims in published maps and institutional affiliations.

Supplementary Information accompanies this paper at (https://doi.org/ 10.1038/s41419-019-1458-8).

Received: 28 November 2018 Revised: 13 February 2019 Accepted: 18 February 2019

Published online: 27 February 2019

\section{References}

1. Tiniakos, D. G., Vos, M. B. \& Brunt, E. M. Nonalcoholic fatty liver disease: pathology and pathogenesis. Annu. Rev. Pathol. 5, 145-171 (2010).

2. Demir, M., Lang, S. \& Steffen, H. M. Nonalcoholic fatty liver disease-current status and future directions. J. Dig. Dis. 16, 541-557 (2015).

3. Fan, J. G. et al. Prevalence of and risk factors for fatty liver in a general population of Shanghai, China. J. Hepatol. 43, 508-514 (2005).

4. Koo, S. H. Nonalcoholic fatty liver disease: molecular mechanisms for the hepatic steatosis. Clin. Mol. Hepatol. 19, 210-215 (2013).

5. Madrigal-Matute, J. \& Cuervo, A. M. Regulation of liver metabolism by autophagy. Gastroenterology 150, 328-339 (2016).

6. Yorimitsu, T. \& Klionsky, D. J. Autophagy: molecular machinery for self-eating. Cell Death Differ. 12(Suppl 2), 1542-1552 (2005).

7. Cuervo, A. M. et al. Autophagy and aging: the importance of maintaining "clean" cells. Autophagy 1, 131-140 (2005).

8. Czaja, M. J. Function of autophagy in nonalcoholic fatty liver disease. Dig. Dis. Sci. 61, 1304-1313 (2016).

9. Martinez-Lopez, N. \& Singh, R. Autophagy and lipid droplets in the liver. Annu. Rev. Nutr. 35, 215-237 (2015).

10. Bojic, L. A. \& Huff, M. W. Peroxisome proliferator-activated receptor delta: a multifaceted metabolic player. Curr. Opin. Lipidol. 24, 171-177 (2013).

11. Tailleux, A., Wouters, K. \& Staels, B. Roles of PPARs in NAFLD: potential therapeutic targets. Biochim. Biophys. Acta 1821, 809-818 (2012).

12. Gross, B., . \& Pawlak, M. \& Lefebvre, P. \& Staels, B. PPARs in obesity-induced T2DM, dyslipidaemia and NAFLD.Nat. Rev. Endocrinol. 13, 36-49 (2016).

13. Francque, S. et al. PPARalpha gene expression correlates with severity and histological treatment response in patients with non-alcoholic steatohepatitis. J. Hepatol. 63, 164-173 (2015).

14. Bajaj, M. et al. Effects of peroxisome proliferator-activated receptor (PPAR)alpha and PPAR-gamma agonists on glucose and lipid metabolism in patients with type 2 diabetes mellitus. Diabetologia 50, 1723-1731 (2007).

15. Musso, G., Cassader, M., Rosina, F. \& Gambino, R. Impact of current treatments on liver disease, glucose metabolism and cardiovascular risk in non-alcoholic fatty liver disease (NAFLD): a systematic review and meta-analysis of randomised trials. Diabetologia 55, 885-904 (2012).

16. Souza-Mello, V. Peroxisome proliferator-activated receptors as targets to treat non-alcoholic fatty liver disease. World J. Hepatol. 7, 1012-1019 (2015).

17. Bays, H. E. et al. MBX-8025, a novel peroxisome proliferator receptor-delta agonist: lipid and other metabolic effects in dyslipidemic overweight patients treated with and without atorvastatin. J. Clin. Endocrinol. Metab. 96, 2889-2897 (2011).

18. Riserus, U. et al. Activation of peroxisome proliferator-activated receptor (PPAR) delta promotes reversal of multiple metabolic abnormalities, reduces oxidative stress, and increases fatty acid oxidation in moderately obese men. Diabetes 57, 332-339 (2008).

19. Tamura, S. \& Shimomura, I. Contribution of adipose tissue and de novo lipogenesis to nonalcoholic fatty liver disease. J. Clin. Invest. 115, 1139-1142 (2005).

20. Mizushima, N., Yoshimori, T. \& Levine, B. Methods in mammalian autophagy research. Cell 140, 313-326 (2010).
21. Sinha, R. A. et al. Caffeine stimulates hepatic lipid metabolism by the autophagy-lysosomal pathway in mice. Hepatology 59, 1366-1380 (2014).

22. Ni, H. M., Bockus, A., Boggess, N., Jaeschke, H. \& Ding, W. X. Activation of autophagy protects against acetaminophen-induced hepatotoxicity. Hepatology 55, 222-232 (2012).

23. Wang, Y. X. et al. Peroxisome-proliferator-activated receptor delta activates fat metabolism to prevent obesity. Cell 113, 159-170 (2003).

24. Toshima, T. et al. Autophagy enhances hepatocellular carcinoma progression by activation of mitochondrial beta-oxidation. J. Gastroenterol. 49, 907-916 (2014).

25. Klionsky, D. J. et al. Guidelines for the use and interpretation of assays for monitoring autophagy. Autophagy 8, 445-544 (2012).

26. Song, Y. M. et al. Dimethyl sulfoxide reduces hepatocellular lipid accumulation through autophagy induction. Autophagy 8, 1085-1097 (2012).

27. Tilg, H. \& Moschen, A. R. Evolution of inflammation in nonalcoholic fatty liver disease: the multiple parallel hits hypothesis. Hepatology 52, 1836-1846 (2010).

28. Lee, J. Y. et al. Dietary oleate has beneficial effects on every step of nonalcoholic fatty liver disease progression in a methionine- and choline-deficient diet-fed animal model. Diabetes Metab. J. 35, 489-496 (2011).

29. Singh, R. Autophagy and regulation of lipid metabolism. Results Probl. Cell Differ. 52, 35-46 (2010).

30. Singh, R. et al. Autophagy regulates lipid metabolism. Nature 458, 1131-1135 (2009).

31. Lin, C. W. et al. Pharmacological promotion of autophagy alleviates steatosis and injury in alcoholic and non-alcoholic fatty liver conditions in mice. J. Hepatol. 58, 993-999 (2013).

32. Song, Y. M. et al. Metformin alleviates hepatosteatosis by restoring SIRT1mediated autophagy induction via an AMP-activated protein kinaseindependent pathway. Autophagy 11, 46-59 (2015).

33. Palomer, X. et al. PPARbeta/delta attenuates palmitate-induced endoplasmic reticulum stress and induces autophagic markers in human cardiac cells. Int. J. Cardiol. 174, 110-118 (2014).

34. Cheng, L. et al. Cardiomyocyte-restricted peroxisome proliferator-activated receptor-delta deletion perturbs myocardial fatty acid oxidation and leads to cardiomyopathy. Nat. Med. 10, 1245-1250 (2004).

35. Mizushima, N., Levine, B., Cuervo, A. M. \& Klionsky, D. J. Autophagy fights disease through cellular self-digestion. Nature 451, 1069-1075 (2008).

36. Bechmann, L. P. et al. The interaction of hepatic lipid and glucose metabolism in liver diseases. J. Hepatol. 56, 952-964 (2012).

37. Komatsu, M. et al. Impairment of starvation-induced and constitutive autophagy in Atg7-deficient mice. J. Cell. Biol. 169, 425-434 (2005).

38. Farah, B. L. et al. Induction of autophagy improves hepatic lipid metabolism in glucose-6-phosphatase deficiency. J. Hepatol. 64, 370-379 (2016).

39. Bojic, L. A. et al. PPARdelta activation attenuates hepatic steatosis in Ldlr-/mice by enhanced fat oxidation, reduced lipogenesis, and improved insulin sensitivity. J. Lipid Res. 55, 1254-1266 (2014).

40. Nagasawa, T. et al. Effects of bezafibrate, PPAR pan-agonist, and GW501516, PPARdelta agonist, on development of steatohepatitis in mice fed a methionine- and choline-deficient diet. Eur. J. Pharmacol. 536, 182-191 (2006).

41. $\mathrm{Wu}, \mathrm{H}$. T. et al. Pharmacological activation of peroxisome proliferator-activated receptor delta improves insulin resistance and hepatic steatosis in high fat diet-induced diabetic mice. Horm. Metab. Res. 43, 631-635 (2011).

42. Kim, J., Kundu, M., Viollet, B. \& Guan, K. L. AMPK and mTOR regulate autophagy through direct phosphorylation of Ulk1. Nat. Cell Biol. 13, 132-141 (2011).

43. Iwaisako, K. et al. Protection from liver fibrosis by a peroxisome proliferatoractivated receptor delta agonist. Proc. Natl Acad. Sci. USA 109, E1369-E1376 (2012).

44. Shan, W. et al. Peroxisome proliferator-activated receptor-beta/delta protects against chemically induced liver toxicity in mice. Hepatology 47, 225-235 (2008). 